PROCEEDINGS OF THE

AMERICAN MATHEMATICAL SOCIETY

Volume 129, Number 1, Pages 45-51

S 0002-9939(00)05496-

Article electronically published on July 27, 2000

\title{
SERIAL SUBALGEBRAS OF FINITARY LIE ALGEBRAS
}

\author{
FELIX LEINEN AND ORAZIO PUGLISI
}

(Communicated by Roe Goodman)

\begin{abstract}
A Lie subalgebra $L$ of $\mathfrak{g l}_{\mathbb{K}}(V)$ is said to be finitary if it consists of elements of finite rank. We show that, if $L$ acts irreducibly on $V$, and if $V$ is infinite-dimensional, then every non-trivial ascendant Lie subalgebra of $L$ acts irreducibly on $V$ too. When Char $\mathbb{K} \neq 2$, it follows that the locally solvable radical of such $L$ is trivial. In general, locally solvable finitary Lie algebras over fields of characteristic $\neq 2$ are hyperabelian.
\end{abstract}

\section{INTRODUCTION}

Let $V$ be a vector space over the field $\mathbb{K}$. The endomorphisms of finite rank form an ideal in $\operatorname{End}_{\mathbb{K}}(V)$, which becomes a locally finite Lie algebra with respect to the usual Lie bracket $[a, b]=a b-b a$ (see [14, p. 32]). We shall denote this Lie algebra by $\mathfrak{f g l}_{\mathbb{K}}(V)$. A Lie algebra $L$ is said to be finitary if there exist a field $\mathbb{K}$ and a $\mathbb{K}$-vector space $V$ such that $L$ is isomorphic to a Lie subalgebra of $\mathfrak{f g}_{\mathbb{K}}(V)$.

The study of finitary Lie algebras is, in part, motivated by the wealth of results available about finitary linear groups, that is, subgroups of $\mathrm{GL}_{\mathbb{K}}(V)$ consisting of elements $g$ such that the endomorphism $g-1$ has finite rank (see [12]). In fact, there is the following relationship between finitary linear groups and finitary Lie algebras. If $G$ is a finitary linear subgroup of $\mathrm{GL}_{\mathbb{K}}(V)$, and if $\mathbb{K} G$ denotes the associative algebra generated by $G$ inside $\operatorname{End}_{\mathbb{K}}(V)$, then the subalgebra

$$
[\mathbb{K} G, \mathbb{K} G]=\sum_{g, h \in G} \mathbb{K}[g, h]=\sum_{g, h \in G} \mathbb{K}[g-1, h-1]
$$

consists of elements of finite rank, so that it becomes a Lie subalgebra of $\mathfrak{f g l}_{\mathbb{K}}(V)$.

Finitary Lie algebras have recently attracted some interest. In [2], 3], 4] simple finitary Lie algebras are investigated. In [13] it is shown that the locally solvable radical of an irreducible Lie subalgebra $L$ of $\mathfrak{f g l}_{\mathbb{K}}(V)$ is trivial whenever $V$ has infinite $\mathbb{K}$-dimension and $L$ is generated by elements of bounded rank. The purpose of this paper is to study serial Lie subalgebras in finitary Lie algebras without specific restrictions on a set of generators.

Received by the editors September 3, 1998 and, in revised form, March 22, 1999.

1991 Mathematics Subject Classification. Primary 17B65, 17B50.

Key words and phrases. Lie algebra, finitary endomorphism, serial subalgebra, locally solvable radical, Hirsch-Plotkin radical. 
Theorem A. Let $L$ be an irreducible Lie subalgebra of $\mathfrak{f g l}_{\mathbb{K}}(V)$, where $\operatorname{dim}_{\mathbb{K}} V$ is infinite.

(a) Let $S$ be a serial Lie subalgebra of L. Either $S$ is nil, or every $S$-composition series in $V$ has finite length, bounded by the smallest rank of a non-nilpotent element in $S$.

(b) Every non-trivial ascendant Lie subalgebra of $L$ acts irreducibly on $V$.

(c) Every locally nilpotent serial (resp. ascendant) Lie subalgebra of L is nil (resp. trivial). In particular, the Hirsch-Plotkin radical of $L$ is trivial.

(d) If Char $\mathbb{K} \neq 2$, then every locally solvable serial (resp. ascendant) Lie subalgebra of $L$ is nil (resp. trivial). In particular, the locally solvable radical of $L$ is trivial.

Note that the situation is quite different for finitary linear groups. The infinite iterated restricted wreath product $C_{p} \mathrm{wr} C_{p} \mathrm{wr} C_{p} \mathrm{wr} \ldots$ of cyclic groups $C_{p}$ of prime order $p$ is a locally nilpotent group which can be represented as a totally imprimitive irreducible finitary linear group over any field of characteristic different from $p$ containing a $p$-th root of unity. In fact, Theorem A shows that infinitedimensional irreducible finitary Lie algebras behave pretty much like non-linear primitive periodic finitary linear groups. There, every non-trivial ascendant subgroup is irreducible [10, Proposition 7.4]. In analogy with the group situation we therefore conjecture that every infinite-dimensional irreducible finitary Lie algebra embeds into the Lie algebra of derivations of a simple finitary Lie algebra (cf. [7] Theorem B]) 1

Theorem B. Let $L$ be a finitary Lie algebra. If Char $\mathbb{K}=2$, let $S$ denote the Hirsch-Plotkin radical in L. Otherwise let $S$ be the locally solvable radical in $L$. Then $S$ is the extension of the largest nil ideal in $L$ by a subdirect sum of finitedimensional Lie algebras. Moreover $S$ is the union of an ascending chain of ideals in $L$ with abelian factors.

Note that even over algebraically closed fields of positive characteristic, nonabelian nilpotent Lie algebras admit irreducible representations of degree larger than one [16].

All notions in our paper are standard and can be found in [1], [5], 15].

\section{Proof of Theorem A}

A Lie subalgebra $S$ of a Lie algebra $L$ is said to be a subideal in $L$ if there exists a finite series $S=S_{0} \leq S_{1} \leq \ldots \leq S_{n}=L$ (where the expression series comprises that each $S_{i}$ is an ideal in $\left.S_{i+1}\right)$. As usual, an $L$-module $V$ is said to have finite length if it has an $L$-composition series with finitely many factors. Recall also that the so-called theorem of Jordan and Hölder applies to modules of finite length.

The proof of Theorem A depends on the following key observation.

Proposition 1. Let $L$ be an irreducible Lie subalgebra of $\mathfrak{f g l}_{\mathbb{K}}(V)$, and let $S$ be a subideal in $L$. If the $S$-module $V$ has finite length greater than one, then $V$ is finite-dimensional, and the $S$-composition factors in $V$ are mutually isomorphic.

Proof. We may assume without loss that $S$ is an ideal in $L$. Because $V$ is an $S$-module of finite length, there exists a minimal $S$-submodule $M$ in $V$. Then

\footnotetext{
${ }^{1}$ Added in proof. This conjecture has been confirmed in the meantime over fields of characteristic zero [8] and over fields of characteristic $p>7$ [9].
} 
$M a \nsubseteq \subseteq M$ for some $a \in L$. The inclusion $M a S \subseteq M S a+M[S, a] \subseteq M a+M$ shows that $M a+M$ is $S$-invariant. Consider the $\mathbb{K}$-epimorphism $\varphi: M \longrightarrow$ $(M a+M) / M$ given by $m \varphi=m a+M$ for all $m \in M$. For every $s \in S$ we have $m s a-m a s=m[s, a] \in M$, whence $\varphi$ is an $S$-epimorphism. Since $M$ is an irreducible $S$-module, $\varphi$ is bijective, and $(M a+M) / M \cong_{S} M$. However, $a$ has finite rank, and so $(M a+M) / M$ and $M$ are finite-dimensional. It follows that $\operatorname{dim}_{\mathbb{K}} M a \leq \operatorname{dim}_{\mathbb{K}} M=\operatorname{dim}_{\mathbb{K}} M a /(M a \cap M) \leq \operatorname{dim}_{\mathbb{K}} M a$. Thus, $M a+M$ is the direct sum of $M a$ and $M$.

Let $M_{1}=M+M L$. The theorem of Jordan and Hölder implies that $M_{1} / M$ is the direct sum of finitely many irreducible $S$-modules $(M a+M) / M$ (where $a \in L$ ) isomorphic to $M$. We now define $M_{i}=M+M L+\ldots+M L^{i}$ and show by induction that $M_{i+1}$ is $S$-invariant and that $M_{i+1} / M_{i}$ is a direct sum of finitely many irreducible $S$-modules $\left(M a_{1} \ldots a_{i+1}+M_{i}\right) / M_{i}$ (where $\left.a_{1}, \ldots, a_{i+1} \in L\right)$ isomorphic to $M$. In the induction step, let $b=a_{1} \ldots a_{i}$ and $a=a_{i+1}$. Suppose that $M b a \nsubseteq M_{i}$. The inclusion $M b a S \subseteq M b S a+M b[S, a] \subseteq M_{i} a+M_{i}$ shows that $M b a+M_{i}$ is $S$ invariant. As above, the $\mathbb{K}$-epimorphism $\left(M b+M_{i-1}\right) / M_{i-1} \longrightarrow\left(M b a+M_{i}\right) / M_{i}$, given by $\left(m b+M_{i-1}\right) \longmapsto m b a+M_{i}$ for all $m \in M$, is an $S$-isomorphism, and the result follows.

Since the $S$-module $V$ has finite length, $V=M_{n}$ for some $n$.

We now aim to reduce our considerations to Lie algebras over algebraically closed fields. The next result can be proved in precisely the same way as its counterpart for finitary linear groups $[6]$.

Proposition 2. Let L be an irreducible Lie subalgebra of $\mathfrak{f g l}_{\mathbb{K}}(V)$, and let $\tilde{\mathbb{K}}$ be a maximal subfield of the division ring $\operatorname{End}_{L}(V)$.

(a) Then $\tilde{L}=\tilde{\mathbb{K}} L$ is an irreducible Lie subalgebra of $\mathfrak{f g l}_{\tilde{\mathbb{K}}}(V)$ satisfying $\operatorname{End}_{\tilde{L}}(V)=$ $\tilde{\mathbb{K}} \cdot \operatorname{id}_{V}$.

(b) $V a$ is $\tilde{\mathbb{K}}$-invariant for every $a \in L$. In particular, the degree of the field extension $\tilde{\mathbb{K}} / \mathbb{K}$ is finite and divides the rank of every $a \in L$.

(c) Let $\overline{\mathbb{K}}$ denote the algebraic closure of the field $\tilde{\mathbb{K}}$, and let $\bar{V}=\overline{\mathbb{K}} \otimes_{\tilde{\mathbb{K}}} V$. Then $\bar{L}=\overline{\mathbb{K}} \otimes_{\tilde{\mathbb{K}}} \tilde{\mathbb{K}} L$ is an irreducible Lie subalgebra of $\mathfrak{f g l}_{\overline{\mathbb{K}}}(\bar{V})$.

In the sequel we shall use the notation introduced in Proposition 2 without further comment. Suppose now that $L$ is an irreducible Lie subalgebra of $\mathfrak{f g l}_{\mathbb{K}}(V)$ and that $\operatorname{dim}_{\mathbb{K}} V$ is infinite. Since every element in $L$ has finite rank, we can choose for every finitely generated Lie subalgebra $F$ of $L$ a finite-dimensional $\tilde{\mathbb{K}}$-subspace $V_{F}$ of $V$ such that $V F=V_{F} F \subseteq V_{F}$. Because $V=V L$, every finite-dimensional subspace of $V$ is contained in one of the so-chosen $V_{F}$.

Proposition 3. For every finitely generated Lie subalgebra $F$ of $L$ there exist a finitely generated Lie subalgebra $F^{*}$ in $L$ containing $F$, and a $\tilde{\mathbb{K}} F^{*}$-composition factor $M_{F} / N_{F}$ in $V_{F}^{*}=V_{F}+V_{F^{*}}$, such that $V_{F} \cap N_{F}=0$ and $V_{F} \subseteq M_{F}$.

Proof. Let $\left\{v_{1}, \ldots, v_{n}\right\}$ be a $\tilde{\mathbb{K}}$-basis of $V_{F}$. Since Proposition 2 implies that $\tilde{L}$ acts densely on $\tilde{V}$, there exist elements $a_{j k}(j, k \in\{1, \ldots, n\})$ in the associative subalgebra $\tilde{\mathbb{K}} \cdot \operatorname{id}_{V}+\sum_{i \geq 1} \tilde{L}^{i}$ of $\operatorname{End}_{\tilde{\mathbb{K}}}(V)$ generated by $\tilde{L}+\tilde{\mathbb{K}} \cdot \mathrm{id}_{V}$, such that $v_{i} a_{j k}=\delta_{i j} v_{k}$ for all $i, j, k$. Choose $F^{*} \subseteq L$ such that the elements $a_{j k}$ are contained in the associative 
subalgebra of $\operatorname{End}_{\tilde{\mathbb{K}}}(V)$ generated by $\tilde{\mathbb{K}} F^{*}+\tilde{\mathbb{K}} \cdot \operatorname{id}_{V} \cdot$ Then

$$
V_{F} \subseteq w\left(\tilde{\mathbb{K}} \cdot \mathrm{id}_{V}+\sum_{i \geq 1}\left(\tilde{\mathbb{K}} F^{*}\right)^{i}\right) \quad \text { for all } w \in V_{F} \backslash 0 .
$$

Since $V_{F}$ is finite-dimensional, there exists a $\tilde{\mathbb{K}} F^{*}$-composition factor $M_{F} / N_{F}$ in $V_{F}^{*}$ such that $M_{F} \cap V_{F} \neq 0=N_{F} \cap V_{F}$. From the above, $V_{F} \subseteq M_{F}$.

Note that, in the situation of Proposition 3, the $F$-module $V_{F}$ embeds into the $F^{*}$-module $W_{F}=M_{F} / N_{F}$ via $V_{F} \ni v \longmapsto v+N_{F} \in W_{F}$.

Using the notation introduced above, we proceed to the proof of Theorem A.

Proof of part (a) of Theorem A. Suppose that $S$ is not nil. Let $s \in S$ be a nonnilpotent element of minimal rank $d$. Assume that $0=V_{0}<V_{1}<\ldots<V_{d+1}=V$ is a $S$-series in $V$. Choose $F=\mathbb{K} \cdot s$. We may assume without loss that $V_{F}$ is large enough so that $V_{i-1} \cap V_{F}<V_{i} \cap V_{F}$ for $1 \leq i \leq d+1$. Then the $\left(V_{i} \cap M_{F}\right)+N_{F} / N_{F}$ $(0 \leq i \leq d+1)$ form a properly ascending $\left(S \cap F^{*}\right)$-series in $W_{F}$. In particular, $W_{F}$ has an $\left(S \cap F^{*}\right)$-composition series of length $\geq d+1$.

Now $S \cap F^{*}$ is a subideal in $F^{*}$. From multiplying each term of a series between $S \cap F^{*}$ and $F^{*}$ with $\tilde{\mathbb{K}}$, we see that $\tilde{\mathbb{K}}\left(S \cap F^{*}\right)$ is a subideal in $\tilde{\mathbb{K}} F^{*}$. Therefore Proposition 1 yields that all $\tilde{\mathbb{K}}\left(S \cap F^{*}\right)$-composition factors in $W_{F}$ are mutually isomorphic. As a $\tilde{\mathbb{K}}$-linear transformation of $V$, the element $s \in S$ is non-nilpotent of $\operatorname{rank} \tilde{d}=d /[\tilde{\mathbb{K}}: \mathbb{K}]$. But $\left(V_{F}+N_{F}\right) / N_{F} \leq W_{F}$, and so any $\tilde{\mathbb{K}}\left(S \cap F^{*}\right)$-composition series in $W_{F}$ has length at most $\tilde{d}$. In order to derive a contradiction it suffices to show that every $\underset{\mathbb{K}}{\mathbb{K}}\left(S \cap F^{*}\right)$-composition factor $U$ in $W_{F}$ has length at most $n=d / \tilde{d}=[\tilde{\mathbb{K}}: \mathbb{K}]$ as an $\left(S \cap F^{*}\right)$-module. But if $U_{0}$ is an irreducible $\left(S \cap F^{*}\right)$ submodule of $U$, and if $\left\{x_{1}, \ldots, x_{n}\right\}$ is a $\mathbb{K}$-basis of $\tilde{\mathbb{K}}$, then $U=x_{1} U_{0}+\ldots+x_{n} U_{0}$ is a direct sum of at most $n$ copies of $U_{0}$.

Proof of part (b) of Theorem A. Let $\left\{S_{\alpha}\right\}_{\alpha \leq \kappa}$ be an ascending series of non-trivial Lie subalgebras in $L$ with $S_{\kappa}=L$, where $S_{\alpha}=\bigcup_{\beta<\alpha} S_{\beta}$ for each limit ordinal $\alpha \leq \kappa$. Choose the smallest $\alpha$ such that $V$ is an irreducible $S_{\alpha}$-module. Assume that $\alpha>0$.

Assume further that $\alpha$ is a limit ordinal. Since $S_{\alpha}$ cannot be nil [13, Lemma 2], there exists $\beta<\alpha$ such that $S_{\beta}$ is not nil. Due to part (a), the $S_{\gamma}$-module $V$ has finite length $d_{\gamma}$ for every $\gamma$ between $\beta$ and $\alpha$. Let $d=\min \left\{d_{\gamma} \mid \beta \leq \gamma<\alpha\right\}$. We may assume that $d_{\gamma}=d$ for $\beta \leq \gamma<\alpha$. It follows that, whenever $\beta \leq \gamma_{1} \leq \gamma_{2}<\alpha$, every $S_{\gamma_{2}}$-composition series in $V$ is a $S_{\gamma_{1}}$-composition series. In particular, if $U_{\gamma}$ denotes the sum of all finite-dimensional irreducible $S_{\gamma}$-submodules in $V$, then $U_{\gamma_{2}} \subseteq U_{\gamma_{1}}$. Since each $U_{\gamma}$ is finite-dimensional, we may assume that $U_{\gamma_{1}}=U_{\gamma_{2}}$ whenever $\beta \leq \gamma_{1} \leq \gamma_{2}<\alpha$. But then $U_{\beta}$ is a finite-dimensional $S_{\alpha}$-submodule in $V$, whence $U_{\beta}=0$. It follows that $V$ contains an infinite-dimensional irreducible $S_{\beta}$-submodule $M$. Since $S_{\gamma}$ is an ideal in $S_{\gamma+1}$, a recursive application of the first argument in the proof of Proposition 1 yields that $M$ is $S_{\gamma}$-invariant for all $\gamma<\alpha$. In particular, $M$ is $S_{\alpha}$-invariant, and $V=M$ is an irreducible $S_{\beta}$-module.

This contradiction to the choice of $\alpha$ shows that $\alpha=\beta+1$ for some $\beta$. But then $S_{\beta}$ is an ideal in the irreducible Lie subalgebra $S_{\alpha}$ of $\mathfrak{f g l}_{\mathbb{K}}(V)$. Hence $S_{\beta}$ cannot be nil [13, Lemma 2]. Now part (a) and Proposition 1 imply that $V$ is finite-dimensional, in contradiction to the hypothesis of Theorem A. 
Proof of part (c) of Theorem A. Because of parts (a) and (b), it suffices to show that a locally nilpotent Lie subalgebra $L$ of $\mathfrak{f g l}_{\mathbb{K}}(V)$ can only act irreducibly on $V$ when $V$ is finite-dimensional. Now $\operatorname{dim}_{\mathbb{K}} V=[\tilde{\mathbb{K}}: \mathbb{K}] \operatorname{dim}_{\tilde{\mathbb{K}}} V=[\tilde{\mathbb{K}}: \mathbb{K}] \operatorname{dim}_{\mathbb{K}} \bar{V}$, and $[\tilde{\mathbb{K}}: \mathbb{K}]$ is finite. We may therefore assume without loss that the field $\mathbb{K}$ is algebraically closed.

Assume that $V$ is infinite-dimensional, and that $L$ acts irreducibly on $V$. Consider $a \in L$, let $F=\mathbb{K} \cdot a$, and choose again $V_{F}$ large enough so that $\operatorname{dim}_{\mathbb{K}} V_{F}$ exceeds the rank of $a$. From [5. p. 41, Corollary of Zassenhaus], every element in $F^{*}$ acts like the sum of a scalar and a nilpotent element on $W_{F}$. By the choice of $\operatorname{dim}_{\mathbb{K}} V_{F}$, the element $a$ induces a singular transformation on $W_{F}$. Thus $a$ acts nilpotently on $W_{F}$, hence also on $V_{F}$ and on $V$. This shows that $L$ is nil, in contradiction to [13, Lemma 2].

Proof of part (d) of Theorem A. As in the proof of part (c), it suffices to show that a locally solvable Lie subalgebra $L$ of $\mathfrak{f g l}_{\mathbb{K}}(V)$ can only act irreducibly on $V$ when $V$ is finite-dimensional. Again we may assume without loss that the field $\mathbb{K}$ is algebraically closed. In the case when Char $\mathbb{K}=0$, such a Lie algebra $L$ has locally nilpotent derived subalgebra $[L, L]$ (see [5 p. 51, Corollary 1]), and hence the assertion follows from part (c). It remains to consider the case when Char $\mathbb{K}=p>2$.

Assume that $V$ is infinite-dimensional, and that $L$ acts irreducibly on $V$. Since $L$ is not nil [13, Lemma 2], we can choose a non-nilpotent element $x \in L$. Let $F=\mathbb{K} \cdot x$, and choose $V_{F}$ large enough so that $\operatorname{dim}_{\mathbb{K}} V_{F}>3 \cdot \operatorname{dim}_{\mathbb{K}} V x$. Let bars denote images in $F^{*}$ modulo the kernel of the representation on $W_{F}$. Replace $\bar{F}$ and $\overline{F^{*}}$ by the smallest $p$-subalgebras of $\mathfrak{g l}_{\mathbb{K}}\left(W_{F}\right)$ containing $\bar{F}$ resp. $\overline{F^{*}}$ (see [15, p. $65])$. In this way, $\bar{F}$ and $\overline{F^{*}}$ become restricted Lie algebras which are still solvable [15, Proposition 2.1.3], and $\operatorname{dim}_{\mathbb{K}} W_{F}>3 \cdot \operatorname{dim}_{\mathbb{K}} W_{F} \bar{x}$. Moreover, the representation of $\overline{F^{*}}$ on $W_{F}$ has character 0 in the sense of [15, p. 210].

Under the hypothesis that Char $\mathbb{K}>2$, it now follows from [15] Theorem 5.8.4] that there is a $p$-subalgebra $Q$ in $\overline{F^{*}}$ and a one-dimensional $Q$-submodule $\mathbb{K} u$ in $W_{F}$ such that

$$
W_{F} \cong \operatorname{Ind}_{Q}^{\overline{F^{*}}}(\mathbb{K} u, 0)=\mathbb{K} u \otimes_{\mathrm{u}(Q)} \mathrm{u}\left(\overline{F^{*}}\right),
$$

where $\mathrm{u}\left(\overline{F^{*}}\right)$, resp. $\mathrm{u}(Q)$, denotes the restricted universal enveloping algebra of $\overline{F^{*}}$ resp. $Q$ (see [15, p. 226, and Sections 5.3 and 2.5]). Let $\left\{e_{n}, \ldots, e_{1}\right\}$ be a $\mathbb{K}$-basis in $\overline{F^{*}}$ such that $\left\{e_{n}, \ldots, e_{m+1}\right\}$ is a $\mathbb{K}$-basis of $Q$ for some $m \geq 1$. From [15, Theorem 2.5.1], the restricted universal enveloping algebras $\mathrm{u}\left(\overline{F^{*}}\right)$ and $\mathrm{u}(Q)$ have $\mathbb{K}$-bases

$$
\left\{e_{n}^{\alpha_{n}} \ldots e_{1}^{\alpha_{1}} \mid 0 \leq \alpha_{i} \leq p-1\right\} \quad \text { resp. } \quad\left\{e_{n}^{\alpha_{n}} \ldots e_{m+1}^{\alpha_{m+1}} \mid 0 \leq \alpha_{i} \leq p-1\right\} .
$$

Hence $\mathbb{K} u \otimes_{\mathrm{u}(Q)} \mathrm{u}\left(\overline{F^{*}}\right)$ has $\mathbb{K}$-basis

$$
\left\{u \otimes e_{m}^{\alpha_{m}} \ldots e_{1}^{\alpha_{1}} \mid 0 \leq \alpha_{i} \leq p-1\right\} .
$$

Assume now that $\bar{x} \notin Q$. We may choose $e_{1}=\bar{x}$. For $0 \leq \alpha_{1} \leq p-2$, the element $\bar{x}$ acts via

$$
\left(u \otimes e_{m}^{\alpha_{m}} \ldots e_{1}^{\alpha_{1}}\right) \cdot \bar{x}=u \otimes e_{m}^{\alpha_{m}} \ldots e_{1}^{\alpha_{1}+1} .
$$

Therefore, as a $\mathbb{K}$-linear transformation of $W_{F}$, the element $\bar{x}$ has rank at least $p^{m-1}(p-1)$, although $\operatorname{dim}_{\mathbb{K}} W_{F}=p^{m}$. This contradicts the choice of $V_{F}$. 
Assume next that $[\bar{a}, \bar{x}] \notin Q$ for some $a \in F^{*}$. In this case we may choose $e_{1}=[\bar{a}, \bar{x}]$, and the above argument shows that

$$
\operatorname{dim}_{\mathbb{K}} W_{F} \leq \frac{3}{2} \cdot \operatorname{dim}_{\mathbb{K}} W_{F}[\bar{a}, \bar{x}] \leq 3 \cdot \operatorname{dim}_{\mathbb{K}} W_{F} \bar{x} .
$$

Again we have a contradiction to the choice of $V_{F}$. Thus, both $\bar{x}$ and $\left[\overline{F^{*}}, \bar{x}\right]$ are contained in $Q$.

For each $\nu \geq 0$, let $U_{\nu}$ be the $\mathbb{K}$-subspace of $\mathbb{K} u \otimes \mathrm{u}(Q) \mathrm{u}\left(\overline{F^{*}}\right)$ generated by $\left\{u \otimes e_{m}^{\alpha_{m}} \ldots e_{1}^{\alpha_{1}} \mid \alpha_{m}+\ldots+\alpha_{1} \leq \nu\right\}$. Since the restricted universal enveloping algebra $\mathrm{u}\left(\overline{F^{*}}\right)$ is a quotient of the universal enveloping algebra of $\overline{F^{*}}$, it follows from [15, Proposition 1.9.1] that $u \otimes \bar{a}_{1} \ldots \bar{a}_{\nu} \in U_{\nu}$ for all $a_{i} \in F^{*}$. Therefore [15. Lemma 5.7.1] yields that, for fixed $0 \leq \alpha_{i} \leq p-1$, the element $\left(u \otimes e_{m}^{\alpha_{m}} \ldots e_{1}^{\alpha_{1}}\right) \cdot \bar{x}$ is congruent to

$$
u \otimes \bar{x} e_{m}^{\alpha_{m}} \ldots e_{1}^{\alpha_{1}}+\sum_{\left\{i \mid \alpha_{i} \geq 1\right\}} \alpha_{i} \cdot u \otimes\left(\left[e_{i}, \bar{x}\right] \cdot e_{m}^{\alpha_{m}} \ldots e_{i+1}^{\alpha_{i+1}} e_{i}^{\alpha_{i}-1} e_{i-1}^{\alpha_{i-1}} \ldots e_{1}^{\alpha_{1}}\right)
$$

modulo $U_{|\alpha|-1}$, where $|\alpha|=\alpha_{m}+\ldots+\alpha_{1}$. Since $\bar{x}$ and $\left[e_{i}, \bar{x}\right]$ are in $Q$, we obtain

$$
\left(u \otimes e_{m}^{\alpha_{m}} \ldots e_{1}^{\alpha_{1}}\right) \cdot \bar{x} \equiv u \bar{x} \otimes e_{m}^{\alpha_{m}} \ldots e_{1}^{\alpha_{1}} \quad \bmod \quad U_{|\alpha|-1}
$$

for $0 \leq \alpha_{i} \leq p-1$. In particular, the sections $\left(\mathbb{K} u \otimes e_{m}^{\alpha_{m}} \ldots e_{1}^{\alpha_{1}}\right)+U_{|\alpha|-1} / U_{|\alpha|-1}$ are mutually isomorphic $\mathbb{K} \bar{x}$-modules. Since $\bar{x}$ acts non-nilpotently on $W_{F}$, we conclude that $\bar{x}$ must act regularly on $W_{F}$, and this is the final contradiction to the choice of $V_{F}$.

The proof of part (d) does in fact reveal the following bound for the ranks of elements in irreducible representations of locally solvable finitary Lie algebras.

Proposition 4. Let $L$ be an irreducible locally solvable Lie subalgebra of $\mathfrak{f g}_{\mathbb{K}}(V)$, and let $d$ denote the rank of a non-nilpotent element in $L$.

(a) If Char $\mathbb{K}=0$, then $d=\operatorname{dim}_{\mathbb{K}} V$.

(b) If Char $\mathbb{K}=p>2$, then $d \geq \frac{p-1}{2 p} \cdot \operatorname{dim}_{\mathbb{K}} V$.

\section{Proof of Theorem B}

Let $L \leq \mathfrak{f g l}_{\mathbb{K}}(V)$, and consider an $L$-composition series $\mathfrak{S}$ in $V$ with factors $U_{\lambda}(\lambda \in \Lambda)$. Let $\rho_{\lambda}: L \longrightarrow \mathfrak{f g l}_{\mathbb{K}}\left(U_{\lambda}\right)$ denote the canonical projection. Then $K=\bigcap_{\lambda \in \Lambda} \operatorname{ker} \rho_{\lambda}$ is the largest nil ideal in $L$ [13. Lemma 2], and Theorem A implies that $\operatorname{dim}_{\mathbb{K}} U_{\lambda}$ is finite whenever $S \rho_{\lambda}$ is non-trivial. Therefore $S$ is the extension of $K$ by a subdirect sum of the finite-dimensional Lie algebras $S \rho_{\lambda}(\lambda \in \Lambda)$.

It follows as in the proof of [13, Proposition 7] that every finite subset of $K$ is contained in an ideal in $L$ which annihilates the factors of a finite series in $V$ whose terms occur in $\mathfrak{S}$ (see also [11, Proof of Theorem B(vi)]). Hence $K$ is the union of an ascending chain of ideals in $L$ with abelian factors. Further $S / K$ is contained in the direct sum of the solvable radicals of those $L \rho_{\lambda}$, for which $U_{\lambda}$ is finite-dimensional, and the intersections of $L / K$ with larger and larger sums of these radicals gives the desired chain in $L / K$.

\section{REFERENCES}

1. R. K. Amayo and I. Stewart, Infinite-dimensional Lie algebras, Noordhoff International Publishing, Leyden, 1974. MR 53:570

2. A. A. Baranov, Diagonal locally finite Lie algebras and a version of Ado's theorem, J. Algebra 199 (1998), 1-39. MR 99f:17026 
3. A. A. Baranov, Simple diagonal locally finite Lie algebras, Proc. London Math. Soc. (3) $7 \mathbf{7}$ (1998), 362-386. MR 99k:17041

4. A. A. Baranov, Finitary simple Lie algebras, J. Algebra 219 (1999), 299-329. CMP 99:17

5. N. Jacobson, Lie algebras, J. Wiley \& Sons, New York - London, 1962. MR 26:1345

6. F. Leinen, Absolute irreducibility for finitary linear groups, Rend. Sem. Mat. Univ. Padova 92 (1994), 59-61. CMP 95:09

7. F. Leinen and O. Puglisi, Countable recognizability of primitive periodic finitary linear groups, Math. Proc. Camb. Phil. Soc. 121 (1997), 425-435. MR 98b:20079

8. F. Leinen and O. Puglisi, Irreducible finitary Lie algebras over fields of characteristic zero, J. Algebra 210 (1998), 697-702. MR 99m:17030

9. F. Leinen and O. Puglisi, Irreducible finitary Lie algebras over fields of positive characteristic, Math. Proc. Camb. Phil. Soc. (to appear).

10. U. Meierfrankenfeld, Ascending subgroups of irreducible finitary linear groups, J. London Math. Soc. (2) 51 (1995), 75-92. MR 96c:20092

11. U. Meierfrankenfeld, R. E. Phillips and O. Puglisi, Locally solvable finitary linear groups, J. London Math. Soc. (2) 47 (1993), 31-40. MR 94c:20064

12. R. E. Phillips, Finitary linear groups: a survey, Finite and locally finite groups (B. Hartley, G. M. Seitz, A. V. Borovik and R. M. Bryant, eds.), NATO ASI Series C 471, Kluwer Academic Publishers, Dordrecht, 1995, pp. 111-146. MR 96m:20080

13. R. E. Phillips and J. Wald, Locally solvable finitary Lie algebras, Comm. Algebra 26 (1998), 4375-4384. MR 99j:17007

14. I. Stewart, Lie algebras generated by finite-dimensional ideals, Pitman Publishing, London San Francisco - Melbourne, 1975. MR 55:12782

15. H. Strade and R. Farnsteiner, Modular Lie algebras and their representations, Marcel Dekker, New York - Basel, 1988. MR 89h:17021

16. H. Zassenhaus, Darstellungstheorie nilpotenter Lie-Ringe bei Charakteristik $p>0$, J. Reine Angew. Math. 182 (1940), 150-155. MR 2:121c

Fachbereich 17 - Mathematik, Johannes Gutenberg-Universität Mainz, D-55099 Mainz, Germany

E-mail address: Leinen@mathematik.uni-mainz.de

Current address: Department of Mathematics, University of Newcastle, Newcastle upon Tyne NE1 7RU, United Kingdom

E-mail address: F.A.Leinen@ncl.ac.uk

Dipartimento di Matematica, Università degli Studi di Trento, I-38050 Povo (Trento), Italy

E-mail address: puglisi@alpha.science.unitn.it 